\title{
The Interactive Effects Of Decision-Making And Expertise On The Experience Of Regret
}

Neel Das, Appalachian State University, USA

Brad Joffe, Citco Fund Services Inc., USA

\begin{abstract}
This empirical research introduces and validates the need for assessing regret from both the decision-making process and the product concurrently, subsequent to an unfavorable decision outcome during post-purchase assessment. Prior regret research in marketing has investigated the experience of regret either from the decision-making process or from the product, but not simultaneously. The research posits and shows that not examining the sources of the regret emotion simultaneously leads to a lop-sided assessment and is likely to inhibit future learning. Results from the study indicate that a differential regret experience is reflected when regret is measured from the decision-making process, however, the same is not revealed when regret is measured from the product.
\end{abstract}

Keywords: Regret; Expertise; Decision Process

\section{INTRODUCTION}

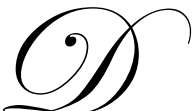

ecisions and decision-making are integral parts of human life. Decisions not only help us choose, inter alia, what we want to do, and when and how to do it, but they also assist us greatly in making subsequent choices. If a decision results in a favorable outcome, we usually experience positive emotions such as happiness, elation, or pleasure. On the other hand, an unfavorable outcome often leads us to experience negative emotions, such as disappointment, frustration, or regret. Of all such negative emotions, regret is a frequently experienced emotion (Shimanoff 1984) and has received the most attention from decision theorists (Connolly and Zeelenberg 2002). Zeelenberg and Pieters (2007) point out that regret, among other things, negatively impacts one's well being and is accompanied by thoughts that one ought to have known better. Overall, the experience of regret is almost never pleasant and it brings about a sinking feeling.
\end{abstract}

Given the importance and the prevalence of this emotion, the aim of this research is to indicate how consumers might be able to learn effectively from a regrettable experience. Prior decisions, more often than not, influence subsequent decisions. In other words, depending on the valence of a current decision outcome, individuals decide how to act and what to do in similar situations in the future. Subsequently, it becomes important to assess properly and learn effectively as to what has happened now, so the same mistakes are not committed in the future. Let us assume that one employs a sub-optimal decision process (like when information search is based on low quality information sources) and consequently chooses a product that turns out to be unfavorable. Upon assessment of what went wrong, if one does not recognize that the decision process was sub-optimal, one is not likely to regret the process and learn from the experience. Hence, to view regret as a futile waste of time is probably a rather myopic view of this commonly if not universally felt emotion (Landman 1993). Regret can be a dynamically changing process that allows individuals to learn from their past mistakes (Landman 1993; Zeelenberg 1999). Saffrey, Summerville, and Roese (2008) found that individuals tend to value their regret experience in positive terms, more so, than they value other negative emotions. This is because people feel that a regret experience is "believed to be beneficial for placing past events in context, preparing to engage in approach and in avoidance behaviors, gaining insight into one's own past behavior and current disposition, and also in facilitating smoother social relations" (p. 52 in Saffrey, Summerville, and Roese, 2008). 
The impetus for this research stems from the idea that traditionally a decision action has been evaluated by assessing the outcome (Higgins 2000). Hence, according to this view, a positive or a favorable outcome evidences a correct decision. However, what if the means for attaining the outcome are unfair? Should that still result in a positive decision assessment? We are familiar with prevalent adages such as "The ends do not justify the means" or "It is not enough to do good, one must do it the right way". The messages derived from such adages refer to the notion that when trying to assess achievements, evaluations should not only concern the final consequence but also how it was attained. Consequently, in terms of decision-making, a decision action will be deemed favorable or positive when both the outcome and the means of achieving the same are proper (Higgins et al. 2008).

The reasoning for the focus of the current paper emanates from the absence of decision assessment (while identifying regret), as discussed in the above paragraph, in prevalent regret research in marketing. Specifically, regret research in marketing has failed to incorporate the assessment of both the decision process and the outcome while measuring regret experienced. Tsiros and Mittall (2000), for instance, developed a model of regret and tested it by means of four studies. However, the items used in measuring regret specifically dealt with product valence. No items were used to measure the decision-making process. Inman and Zeelenberg (2002), on the other hand, focused essentially on the decision process in their investigation of whether regret experienced is greater from maintaining a status quo decision or from deciding to switch. Bui, Krishen, and Bates (2011) in their investigation of the effects of regret on brand switching intentions, satisfaction, and ruminative thoughts have assessed regret from the point of view of the outcome (or product) only. Cai and Cude (2011), in their research on the effects of the presence or absence of reference prices on the experience of regret, only assessed regret from the decision. It is obvious that our understanding of the regret emotion has been advanced tremendously due to the research as identified above. However, we feel that our current conceptualization of regret measurement would further aid in better understanding the experience of the emotion.

In line with the aim and the reasoning for the focus of this research, the specific objective here is to introduce the notion that subsequent to experiencing regret, it is important to measure the emotion from both the decision-making process and the outcome (or product, for consumer decision making) concurrently. In other words, while evaluating a decision action, it becomes imperative to verify whether and how regret might arise from the decision process (that is, how a decision is made) and/or product (that is, the product purchased due to a decision process). We endeavor to show that assessing regret only from the decision process or the product is likely to provide a lop-sided view of the emotion. On the other hand, a concurrent examination is likely to show any misattribution (like regretting the process (product) when the product (process) is actually regrettable) that might occur. If the decision process (or the outcome) is regrettable but individuals do not regret the same, they are not likely to learn from their mistake and may commit the same error in future.

\section{REGRET: A CONCEPTUAL AND THEORETICAL FRAMEWORK}

Loomes and Sugden (1982) introduced the notion of regret theory as an explanation of rational behavior under uncertainty and suggested it to be a simpler alternative to prospect theory offered by Kahneman and Tversky (1979). According to Loomes and Sugden (1982), regret is experienced when consumers believe that a different choice would have led to a better outcome (compared to the one received from the current choice), even though the initial choice was the best alterative at the time it was made. The following discussion presents the conceptualization of regret by Loomes and Sugden (1982). All nomenclatures used in the discussion are adapted from Loomes and Sugden (1982).

If there is a choice between two actions $\left(\mathrm{A}_{1}\right.$ and $\left.\mathrm{A}_{2}\right)$, individuals are likely to choose an action $\left(\mathrm{A}_{1}\right.$ or $\left.\mathrm{A}_{2}\right)$ that maximizes their expected utilities. Such an assumption of choice exists since regret in theory is an explanation of rational behavior under uncertainty. When an individual chooses one action (say $\mathrm{A}_{1}$ ), he/she experiences a consequence $\left(\mathrm{x}_{1 \mathrm{j}}\right)$, where ' $\mathrm{j}$ ' is the state of world that occurs due to the choice. There are ' $\mathrm{n}$ ' possible states of the world $\left(\mathrm{S}_{1}, \mathrm{~S}_{2}, \ldots \mathrm{S}_{\mathrm{n}}\right)$, however, individuals are not sure about which one may occur at the time of making a choice. The resultant psychological experience of (dis)pleasure occurring from the choice depends not only on the nature of the consequence of choosing $A_{1}$, but also on the nature of consequence if one had chosen $A_{2}$ (i.e. $x_{2 j}$ ). Hence, if $A_{1}$ were the better decision, one would experience rejoicing; alternatively, if $\mathrm{A}_{2}$ were the better decision, one would experience regret. When the psychological experience (of regret or rejoicing) occurs this way, it is assumed to be 
independent of any other characteristic of the consequences. In other words, the psychological experience is strictly based on a comparison of 'what is' and 'what might have been'. Consequently, when 'what is' compares to 'what might have been' there will be neither regret nor rejoicing. Additionally, the more pleasurable 'what might have been' is, the greater is the regret experienced. This is the fundamental idea of regret theory as introduced by Loomes and Sugden (1982).

Following the pioneering work by Loomes and Sugden (1982), various researchers in psychology and marketing have since investigated and expanded our knowledge regarding the regret emotion. The following discussion provides some examples that show how research in psychology has advanced our understanding of regret. Sugden (1985) proposed that along with the wish that one had chosen differently, the notion of selfrecrimination ought to be incorporated as another component of regret. Landman (1993), in her treatise on regret carefully dissected the emotion to show, inter alia, how regret (although conceptualized as an emotion) included a high amount of cognition. Gilovich and Medvec (1995) reiterated Landman's (1993) position and further stated that regret stems from two sources: errors of commission (action) and omission (inaction) and found that while action regrets decrease, inaction regrets increase over time. Zeelenberg et al. (2002) identified that under certain conditions, like when considering a sequence of past decisions in order to contemplate what to do in the current time, inaction regrets were greater than action regrets. Zeelenberg and Pieters (2007) proposed the theory of regret regulation wherein they suggested that individuals actively chose alternatives that would result in minimizing the resultant regret due to being regret adverse.

Within the domain of marketing, Simonson (1992) examined the role of anticipated regret and showed that if consumers are made to anticipate the possible decision errors with regard to buying an item now as opposed to waiting for a better deal, they usually made the choice of buying the item in the current period. Subsequently, Taylor (1997) posited that expectations about 'unchosen' alternatives are likely to have an effect on one's choice, especially when the expectations regarding the chosen alternative were not met. Inman, Jia, and Dyer (1997) extended the work of Taylor (1997) by incorporating disappointment and regret in the model of satisfaction. Tsiros and Mittal (2000) developed a model of experienced regret, presented below in Figure 1, and established a multi-item scale for measuring the emotion. The model (below) developed by Tsiros and Mittal (2000) represents a landmark in regret research in marketing in terms of identifying the antecedent, moderators, and consequences of the felt emotion. The antecedent of regret is identified as the information on the forgone outcome. In that, even if individuals are not aware of what they have missed, regret might still be experienced because of counterfactuals or hypothetical scenarios that are constructed by the consumers. These scenarios provide the consumers with a basis of comparison when the information on the forgone outcome is missing. Understandably, the intensity of regret is likely to be lower when individuals construct such counterfactuals as opposed to when the information is present.

However individuals do not always construct counterfactuals in their minds. The motivation to generate these scenarios depends on three situation-specific characteristics - status quo, reversibility of the outcome and the valence of the outcome. These are included in the model as moderators affecting the relationship between knowledge of the forgone outcome and the regret experienced. The consequences of regret are repurchase intentions, satisfaction and complaint intentions.

Regret research in marketing has also examined the effects of the emotion within the context of other marketing variables. Bolton, Kannan and Bramlett (2000), include the notion of regret to examine loyalty reward programs and find that loyal consumers tend not to regret the price disadvantages and the overall quality ratings of the company they are loyal to compared to similar assessments of other companies. Syam, Krishnamurthy and Hess (2008) looked at the preference structures for customized versus standard products and showed that when individuals tend to become regret averse their inclination towards standard (versus customized) products increase. Cai and Cude (2011) looked at regret on an online bidding task within the context of absence or presence of reference prices. They concluded that subsequent to a negative outcome, the presence (vis-à-vis the absence) of reference prices induced more regret. 


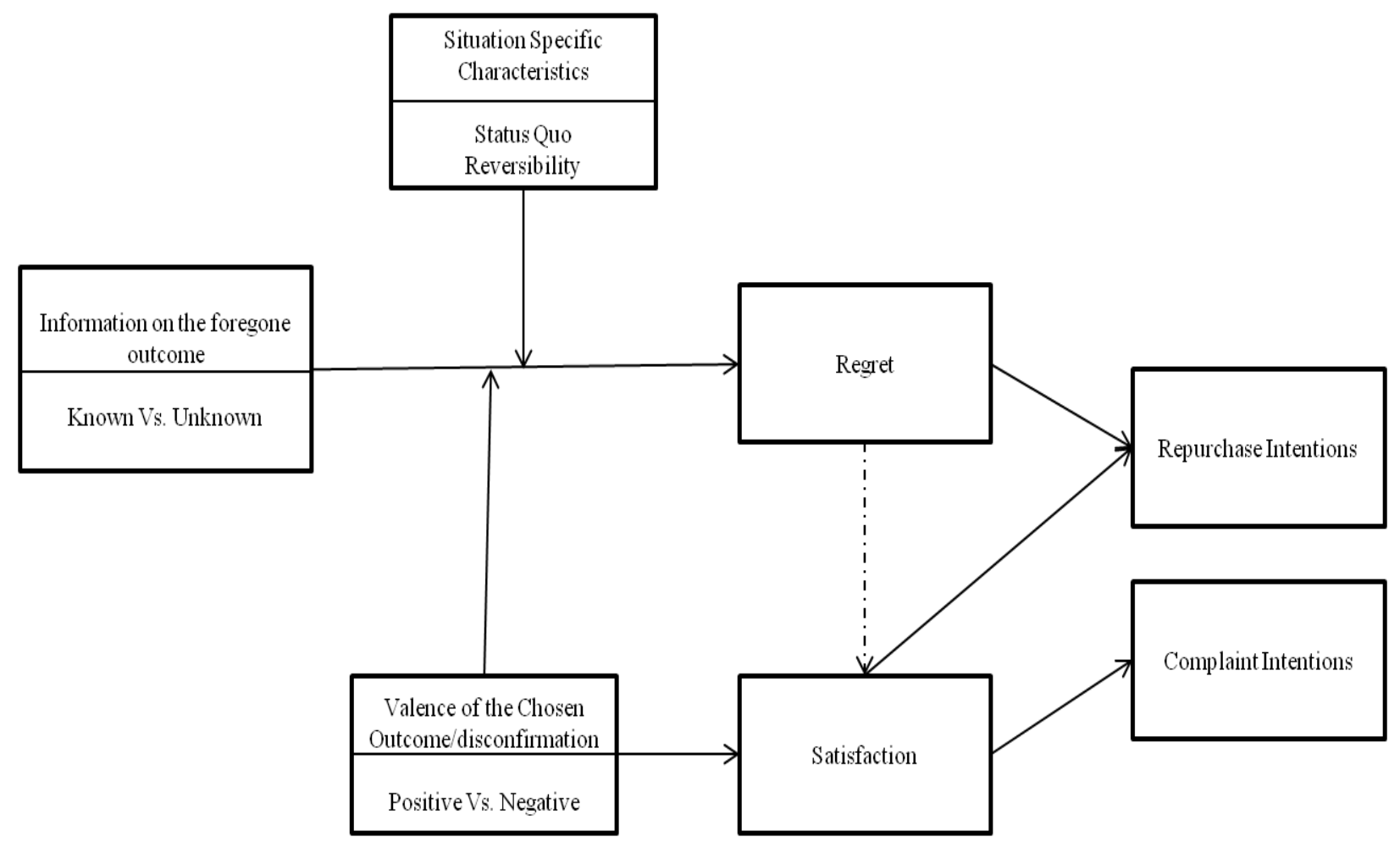

Figure 1: A Model of Regret in Consumer Decision-Making (Adapted from Tsiros and Mittal 2000)

\section{LITERATURE REVIEW}

Heitmann, Lehmann, and Herrmann (2007) investigate the notions of consumption and decision satisfaction as separate constructs determining overall consumer satisfaction. These authors posit that the anticipation of regret affects the decision satisfaction, which in turn affects consumption satisfaction leading to behavioral implications of loyalty, product recommendations, and word-of-mouth. In developing their model, they also mention "traditionally, satisfaction has been conceptualized as a product-related judgment" (p. 234). Such a focus on two types of satisfaction underlines the objective of this current research. As mentioned earlier, researchers such as Taylor (1997) and Inman, Jia, and Dyer (1997) endorse the inclusion of regret, along with satisfaction, for an optimal post choice evaluation. However, if regret is conceived and measured only from the point of view of a decision or a product, it is unlikely to achieve such an optimal assessment. In other words, stemming from Heitmann et al.'s research, intuitively it appears that regret be assessed in a similar fashion.

So what makes an optimal decision? An optimal decision is one where the outcome derived is valuable and worthwhile and also when the means used to achieve the outcome is right or proper (Higgins 2000). Alternatively, a regrettable decision is a combination of regret arising from the outcome (when compared against a rejected outcome) and regret arising from an unjustified decision process (Connolly and Zeelenberg 2002, Pieters and Zeelenberg 2005). Such a conceptualization of a regrettable decision, termed Decision Justification Theory, was contemplated in psychology by Connolly and Zeelenberg (2002). Prior to that, regret research in marketing and psychology have essentially measured regret either from an outcome or from a decision-process.

To provide further salience to our objective regarding the need for assessing the components of regret in order to provide functionality and benefit learning, we look at two vignettes provided by Sugden (1985):

You choose to make a journey by car rather than by train. You are involved in an accident through no fault of your own. If you had taken the train you would have avoided the accident. You may regret your decision to go by car- 
that is, you may wish that you had gone by train - even though there was no way you could have known in advance that going by train would turn out better (p. 78).

You choose to drive home rather than take a taxi after you have had too much to drink. You don't have an accident and you are not stopped by the police. Next morning you may regret the foolish risks that you ran, even though you came to no harm and saved a taxi fare (p. 79).

While the first situation indicates regret arising essentially due to the outcome, the second situation indicates regret due to a sub-optimal decision process. In the first vignette, the sooner one realizes that it is not his/her fault, the easier it will be to move on; alternatively, if the decision-making process of choosing to make the journey by car is regretted, then such rumination is likely to turn into an exercise in lamentation. On the other hand, if the decision-making process is not regretted in the second vignette and only the final outcome is evaluated, then it will clearly be dysfunctional since it may prompt one to be more foolhardy in the future, thereby not only risking one's own life but also of others.

Within a consumer decision-making context we believe that a sub-optimal decision-making process resulting in a fortuitous favorable product choice is not likely to motivate individuals to conduct a post-decision assessment. Hence, for our present purposes we consider a situation when the product choice is regrettable and the decision-making process is either optimal (i.e. good) or sub-optimal (i.e. bad). A sub-optimal decision-making process occurs when, for instance, information search regarding and evaluation of alternatives is conducted less intensively and/or is based on low quality information sources (Janis and Mann 1977). On the other hand, the product purchased is regrettable when the consumer learns, post-purchase, about the existence of a better performing (compared to the chosen) alternative in the marketplace. The assumption in terms of the product purchased is that a consumer does not knowingly forgo a better performing product. Hence, s/he chose the product that at the time of making the decision appeared to be the 'right' one.

To the best of our knowledge, the primary research within the marketing domain to introduce the idea of regret arising from a decision process and outcome is by Das and Kerr (2010). In their conceptual research, they introduce the notion of regret arising from two sources and posit the role of two moderating variables - need for cognition and regulatory focus, in terms of how they might enhance the understanding of the sources of regret from an individual perspective. We extend that research in terms of an empirical examination and include the notion of the expertise of the consumer as a moderator.

Research in cognitive psychology delineate that expertise is domain related, i.e. special skills of experts are absent outside the area of one's expertise (Shanteau 1992). A novice or a non-expert individual is one who lacks the requisite skills and abilities to perform in a specific area. Within the context of our current research, we adopt this particular view of experts/non-experts. Of the different psychological features present in an expert, one particular characteristic is especially important for us - experts have a strong sense of responsibility (Shanteau 1992). Responsibility has a positive association with regret, with greater responsibility determining greater regret (Zeelenberg, Van Dijk, and Manstead 1998, 2000). In other words, the notion of personal agency is key to the experience of regret. Hence, if one is responsible for the decision that one makes, subsequent to a poor outcome due to the decision made, one is likely to experience regret from that decision. Alternatively, if one is not responsible enough for one's decision, regret experienced is likely to be less, subsequent to an unfavorable outcome. Hence, we hypothesize that

H1: When the decision-making process is unfavorable, subsequent to an unfavorable outcome, experts will experience greater regret than non-experts.

On the other hand, when the product is regrettable, a different story emerges. First, let us revisit our definition of a regrettable product. A regrettable product means getting to know, post-purchase, about the existence of a better performing (compared to the chosen) product in the marketplace/space. Although, experts have superior knowledge of available alternatives (Mitchell and Dacin 1996) and the requisite knowledge to choose a particular product for a specific usage situation (Brucks 1985), it is relatively straightforward to assume that no one has complete information concerning the entire gamut of choices available. Hence, when an expert becomes aware of a 
better performing product, he/she might feel less responsible and experience less regret. After all, the existence of a better-performing product could be a matter of chance or luck. However, given that non-experts are less confident (relative to experts) of their decision-making process, they are less likely to believe that the presence of a foregone better performing product is due to chance. Probably they could be more likely to blame themselves for not conducting a thorough search of the available alternatives and hence feel more responsible for missing out on a better product. Hence, we hypothesize that

H2: When the product is unfavorable, subsequent to an unfavorable outcome, non-experts will experience greater regret than experts.

\section{METHODOLOGY}

\section{Method}

A pre-test was conducted to find out what might be a suitable product for our student sample and to indentify good and bad decision-making process in terms of information search. The pre-test was administered to 20 students. The results of the pre-test indicated the following products for which students are likely to think in terms of purchasing and also think after purchasing, in case of an unfavorable outcome due to a purchase - car, house, computer, and vacation. We decided to use the product category of cars for our main study.

In terms of favorable information gathering, the following were identified (for buying a car):

1. Reviews in Consumer Reports and Carfax.

2. Test-driving.

3. Opinion of friends and family members who have owned similar cars.

4. Research regarding other alternatives.

In terms of unfavorable information gathering, the following were identified (for buying a car):

1. How many people drive the cars that match your needs.

2. Prices of cars.

3. $\quad$ Ads noticed.

4. Opinion of the salesperson.

The main study used a 2 (decision-making: good vs. bad) X 2 (expertise: high vs. low) between subjects design where expertise was manipulated as a self-reported measure based on previous research (Mitchell and Dacin 1996).

\section{Participants, Procedure, and Data Collection}

Data was collected in a computer lab from eighty undergraduate business students (43 females; median age $=21.2$ years) in a mid-size university in the southeast. Students participated in the study for course credit. Upon entering the lab, participants were greeted by the experimenter and seated in front of a laptop computer. The experimenter then asked them to carefully read and answer the questions that appeared on the computer screen. The first 3 questions (on a 7-point scale) were asked to evaluate a decision action was assessed: (1) "When assessing a decision you have made, how important is it for the decision-making process to be optimum?" (1: not at all / 7: very much); (2) When assessing a decision you have made, how important is it for the product to work well?" (1: not at all / 7: very much); (3) When assessing a decision you have made, which part of the decision is more important" (1: decision-making process / 7: product).

The objective of the above questions was to find out whether decision evaluation is traditionally made (i.e. based on the valence of the product) or whether both the process and product is assessed equally. Following these questions, respondents were generally asked about their expertise regarding cars. These questions, the items for which are detailed below, constituted the expertise of the respondents. The reason for measuring expertise without having the students be exposed to the scenario was to make ensure that any bias arising after having read the 
scenario would be avoided. After the expertise questions, respondents exposed to a distraction task (for about 2 minutes) where they were asked to identify several celebrities from different movie clips.

Following the distraction task, the scenarios were instituted where the respondents were asked to imagine that they were shopping for a car. Students in the optimal decision-making condition read:

You have been gathering information about the cars that will match your needs based on: The reviews of the cars on Consumer Reports.org and Carfax.com.; How the cars felt when you test drove them; What your friends and family said who have owned similar cars; The research you have done to fully evaluate other alternatives.

Students in the sub-optimal decision-making condition read:

You have been gathering information about the cars that will match your needs based on: How many people you see drive the cars that may match your needs; Prices of cars; The ads noticed you have noticed; Opinion of the salesperson.

Subsequent to the above decision-making manipulation they were told to imagine that they had decided to buy Ford Focus, while a friend who was also in the market to buy a car decided to buy a Nissan Sentra. Finally, to ensure that the participants experienced regret from the product (i.e. Ford Focus) they were given the following information:

Now, after a few weeks, you are continually running into problems with your Ford Focus. The car has already been to the shop for a malfunctioning power window and the needs-maintenance light, on the dash, keeps turning on and off. The audio system in the car does not function properly and keeps randomly shutting off. On several occasions you could not even start the car. Moreover, the service at the dealership has been terrible. Your friend, on the other hand, does not have any problems with the Nissan Sentra.

\section{Measures}

Expertise was manipulated as a self-reported measured variable on a 7-point scale using the following items (Mitchell and Dacin 1996): (1) "How familiar are you with cars" (very unfamiliar, very familiar); (2) "How clear an idea do you have about which characteristics are important in providing you usage satisfaction" (very unclear, very clear); "I know a lot about car" (strongly disagree, strongly agree); "How would you rate your knowledge about cars relative to the rest of the population (Very unknowledgeable, very knowledgeable). The items were averaged (coefficient alpha $=.88$ ) and a median split was used to separate the high from low expertise participants.

The dependent variables of regret from the product and regret from decision-making were adopted from Tsiros and Mittal (2000). Regret due to the decision-making process was assessed by having respondents agree/disagree with the following three statements: (1) "You feel sorry regarding the process you used in gathering information"; (2) "You regret the process you used in gathering information"; (3) "You should have used a different process in gathering information" (strongly disagree (1)/strongly agree (7). The items were averaged to create a Process-based regret index (coefficient alpha $=.90$ ). Regret due to product was similarly measured by asking the respondents the extent to which they agreed with the following three statements (1) "You feel sorry for choosing the Ford Focus"; (2) "You regret choosing the Ford Focus"; (3) "You should have chosen the Nissan Sentra" (anchors: strongly disagree (1)/strong agree (7)). The items were averaged to create a Product-based regret measure (coefficient alpha $=.70)$.

\section{Manipulation Checks}

Manipulation checks were performed for the decision-making variable with the following questions: (1) overall how would you rate your experience (1: unfavorable, 7: favorable); (2) how likely is it that your overall experience is due to the information gathering process (1: very unlikely, 7: very likely); (3) how likely is your overall experience due to the product (1: very unlikely, 7: very likely); (4) how would you rate the quality of your 
information gathering process (1: unfavorable, 7: favorable); (5) how would you rate the quality of the product (1: unfavorable, 7: favorable). All the questions were on a $1-7$ scale.

\section{RESULTS}

A paired sample $t$-test indicated that when assessing a decision it was significantly more important for the product to work well $\left(\mathrm{MEAN}_{\text {PRODUCT }}=6.61\right)$ than the decision-making process to be optimum $\left(\mathrm{MEAN}_{\mathrm{DECISION}}\right.$ PROCESS $\left.=5.63 ; t_{79}=-7.74, \mathrm{p}<.001\right)$. In terms of whether the decision-making process vis-à-vis the product was more important while assessing a decision, the mean was 4.71 and the median was 5.00.

Independent sample $t$-tests provided proof that our manipulation for good versus bad decision-making was understood as intended. The results for the manipulation checks are provided in Table 1 below:

Table 1: Manipulation Checks

\begin{tabular}{|l|c|c|c|}
\hline \multicolumn{1}{|c|}{ Manipulation Questions } & Means: Bad Decision & Means: Good Decision & $\mathbf{t}_{(\mathbf{7 8})}$ value \\
\hline $\begin{array}{l}\text { Overall experience } \\
\text { (1: unfavorable / 7: favorable) }\end{array}$ & 1.87 & 1.95 & -0.27 \\
\hline $\begin{array}{l}\text { Experience due to information gathering (1: very } \\
\text { unlikely / 7: very likely) }\end{array}$ & 4.90 & 3.20 & $5.64^{*}$ \\
\hline $\begin{array}{l}\text { Experience due to product } \\
\text { (1: very unlikely / 7: very likely) }\end{array}$ & 4.87 & 4.54 & 0.96 \\
\hline $\begin{array}{l}\text { Quality of information gathering process } \\
\text { (1: unfavorable / 7: favorable) }\end{array}$ & 3.08 & 4.83 & $-6.06^{*}$ \\
\hline $\begin{array}{l}\text { Quality of product } \\
\text { (1: unfavorable / 7: favorable) }\end{array}$ & 2.00 & 2.05 & -0.20 \\
\hline
\end{tabular}

$* \mathrm{p}<.001$

A 2 X 2 MANOVA on regret due to decision-making and product revealed a significant multivariate interaction $(\mathrm{F}=4.14, \mathrm{p}<.05)$. The multivariate interaction was due to the univariate interaction on decision making process $(\mathrm{F}=8.35, \mathrm{p}<.01)$. The multivariate and univariate effects of decision-making and expertise on regret due to decision-making and product are illustrated in Table 2 below:

Table 2: Multivariate and Univariate Effects of Decision Making type and Expertise on Regret due to Decision-Making and Product

\begin{tabular}{|l|c|c|c|c|}
\hline \multicolumn{1}{|c|}{ Effect } & \multicolumn{2}{c|}{ Multivariate } & \multicolumn{2}{c|}{ Univariate } \\
\hline & & & Process & Product \\
\cline { 3 - 5 } & Wilk's Lambda & F- Value & F- Value & F- Value \\
\hline Expertise & 0.931 & 2.79 & 3.45 & 2.08 \\
\hline Decision Making & 0.553 & $30.33^{* * *}$ & $60.50^{* * *}$ & 0.66 \\
\hline Expertise X Decision Making & 0.901 & $4.14^{*}$ & $8.35^{* *}$ & 0.02 \\
\hline
\end{tabular}

$* * * \mathrm{p}<.001 ; * * \mathrm{p}<.01 ; * \mathrm{p}<.05$

According to $\mathrm{H} 1$, when the decision-making process was unfavorable (i.e. a sub-optimal decision process condition), subsequent to an unfavorable outcome, experts would experience greater regret than non-experts. As can be seen from Figures 1 (measuring regret from decision-making) and 2 (measuring regret from product) below, this hypothesis was not supported. Specifically, when measuring regret from decision-making process, a sub-optimal decision process elicited similar intensities of regret across experts $(\mathrm{MEAN}=5.47)$ versus non-experts $(\mathrm{MEAN}=$ $5.22 ; t_{37}=-0.80$, n.s.). Similarly, when measuring regret from product, a sub-optimal decision process elicited similar intensities of regret across experts (MEAN $=5.70)$ versus non-experts $\left(\mathrm{MEAN}=6.07 ; t_{37}=0.39\right.$, n.s. $)$. Hence, H1 was not supported regardless of whether regret was measured in terms of decision-making process or product. 
According to $\mathrm{H} 2$, when the product was unfavorable (i.e. optimal decision process condition), subsequent to an unfavorable outcome, non-experts would experience greater regret than experts. As can be seen from Figure 1 (i.e. when measuring regret from decision-making) an unfavorable product resulted in significantly greater regret for non-experts $(\mathrm{MEAN}=4.00)$ than for experts $\left(\mathrm{MEAN}=2.82 ; t_{39}=3.15, \mathrm{p}<0.01\right)$. Alternatively, when measuring regret from product, an unfavorable product elicited similar intensities of regret across experts $(\mathrm{MEAN}=5.44)$ versus non-experts $\left(\mathrm{MEAN}=5.87 ; t_{39}=1.19\right.$, n.s.). Hence, $\mathrm{H} 2$ was supported when regret was measured in terms of the decision-making process, but not in terms of product.

The means table for the above two hypotheses is presented below in Table 3:

Table 3: Means Table

\begin{tabular}{|c|c|c|c|c|}
\hline Measures of Regret & Sub-optimal Decision & $t$ & Optimal Decision & $t$ \\
\hline Regret due to Decision-Making & $\begin{array}{l}\text { Expert: } 5.47 \\
\text { Non Expert: } 5.22\end{array}$ & -0.80 & $\begin{array}{l}\text { Expert: } 2.82 \\
\text { Non Expert: } 4.00\end{array}$ & $3.15^{*}$ \\
\hline Regret Due to Product & $\begin{array}{l}\text { Expert: } 5.70 \\
\text { Non Expert: } 6.07\end{array}$ & 0.39 & $\begin{array}{l}\text { Expert: } 5.44 \\
\text { Non Expert: } 5.87\end{array}$ & 1.19 \\
\hline
\end{tabular}

$* \mathbf{p}<.01$

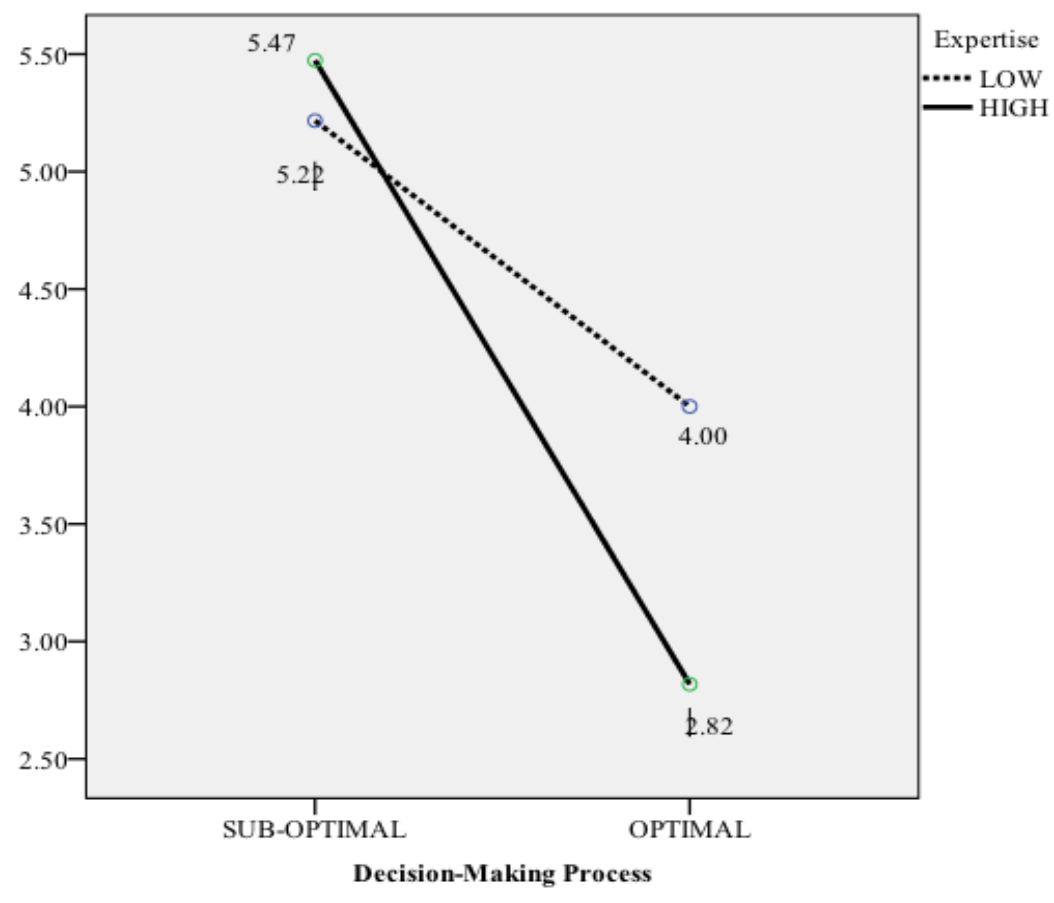

Figure 1: Univariate interaction of Decision-Making Conditions and Expertise on Regret from Decision -Making Process 


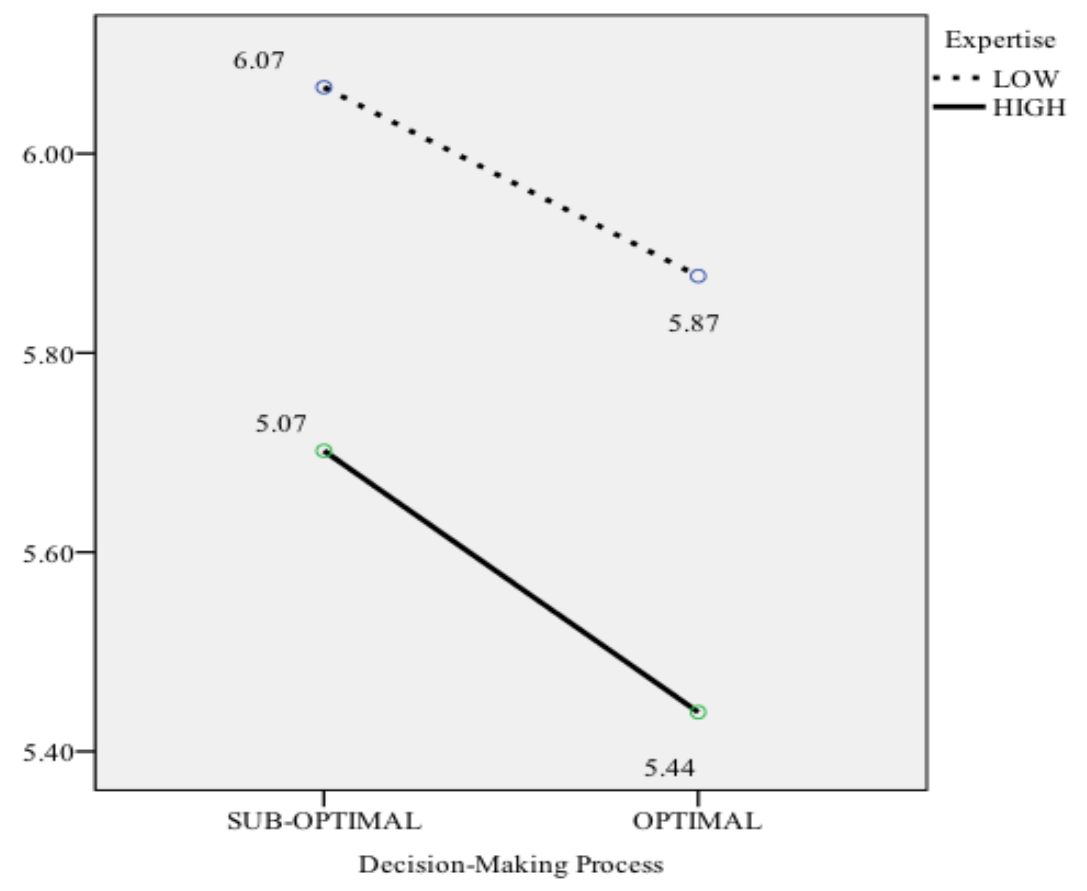

Figure 2: Univariate interaction of Decision-Making Conditions and Expertise on Regret from Product

\section{DISCUSSION}

The results of the study provide some interesting insights into the process of assessment of a regrettable decision action. First, as can be observed from the paired sample $t$-test of the decision evaluation questions, respondents indicated that it was more important that a product performs well compared to the importance of the optimality of a decision-making process. This finding underlines what researchers have established earlier in that a decision outcome is evaluated primarily in terms of product performance than the analysis of the decision process (e.g. Higgins 2000; Heitmann, Lehmann, and Herrmann 2007). The median score further validated such dominance of product performance when asked specifically which part of the decision made was important (1: decision-making process / 7: product) when evaluating a decision. However, this does not imply that the decision-process is not important during an evaluation - the results reiterate that both (i.e. product performance and optimality of decisionmaking process) are important, but the product performance is significantly more important. This then provides the foundation, as explained earlier, on which we built our research objective that a simultaneous examination of regret from decision-making process and product would likely provide a better explanation of the emotion experienced.

Second, the moderating effects of expertise on regret experienced were observed differentially depending on whether regret was measured from the decision-making process or from the product. Results did not support our initial proposition that experts would experience greater regret than non-experts for a sub-optimal decision process (leading to an unfavorable product choice). Experts and non-experts, regardless of whether regret was measured from the decision process or the product, regretted a sub-optimal decision process equally. We had, however, posited that due to greater responsibility on the part of experts they would experience greater regret. An explanation of this anomaly may be that non-experts when thinking about 'what might have been' in terms of a decision process are able to comprehend when a process implemented was sub-optimal. In other words, within the context of our research it is difficult to argue that non-experts do not feel responsible for the implementation of a sub-optimal decision process. Such a finding probably provides an interesting boundary condition to what Shanteau (1992) had advised earlier that experts usually possess a strong sense of responsibility. Based on the results of our study we forward the notion that such responsibility is likely to be universally experienced by experts and non-experts alike. 
Non-experts, on the other hand, regretted the optimal decision process more subsequent to an unfavorable outcome, when regret was measured from the decision process. This result follows our prediction that non-experts, given their paucity of knowledge vis-à-vis experts, probably fail to recognize an optimal decision-process. Such failure is made even more salient when the decision process leading to the outcome is unfavorable. On the other hand, the enhanced knowledge structure of the experts helps them to recognize that the decision process implemented was optimal and the failure of the product was probably more of a chance rather than their own fault. This finding also extends prior research on expertise where scholars point out that laypersons consistently assess decision quality in terms of outcomes (Yates and Tschirhart 2006). Hence, non-experts are likely to experience greater regret even from an optimal decision process when the product is unfavorable since they are likely to anchor their assessment in terms of the product.

In addition to the above, interestingly the support for our second hypothesis was obtained when assessing regret from the decision process but not when assessing regret from the product. This then highlights the objective of our study that subsequent to experiencing regret, it is important to measure the emotion from both the decisionmaking process and the outcome (or product, for consumer decision making) concurrently. Specifically, if regret were measured only from the product we would have failed to identify the interactive effects of expertise and decision-making conditions on the experience of regret. On the other hand, if regret were measured only from the decision-making process, we would have failed to observe that subsequent to an unfavorable outcome from a suboptimal decision process, both experts and non-experts are likely to experience equivalent intensities of regret. Hence, the study validates our assumption that assessing regret only from one source probably would provide a lopsided view of the emotion. The current research also provides the following theoretical contributions as noted:

- It is important to evaluate regret from both the decision-making process and the product concurrently to obtain a holistic view of the emotion.

- The activation of regret due to different sources is also likely to result in different behavioral intentions. For instance, Tsiros and Mittal (2000) concluded that regret has a direct inverse influence on re-purchase intentions, in that, greater the regret lower the re-purchase intentions. However, if regret arises from one's decision-making process, then one is likely to attribute the blame to one's self and hence might not express lower re-purchase intentions while experiencing greater regret. Zeelenberg and Pieters (2004), in the context of services, showed that regret has a direct positive relationship on switching intentions. However, when the one's decision-making process is regretted, one might not always exhibit an intention to switch, given that more self-blame is likely to be employed.

- A concurrent assessment may also help in better understanding whether and how consumers learn from their regrettable experiences. For instance, Ratner and Herbst (2005) have shown that emotional reactions subsequent to unfavorable outcomes sometimes lead individuals to adopt a less than optimal decision the next time they purchase a product, even though the foregone decision has a better chance of producing a better outcome. How is this likely to happen? According to our research if experts and non-experts assess regret solely from the product, they might mis-forecast the worth of their decision process when it is optimal and thereby change it the next time around. This is likely to impede learning.

- $\quad$ Das and Kerr (2010) had conceptualized the two sources of regret using need for cognition and regulatory focus. We implemented an empirical study and included the expertise of consumers to further elucidate the idea and the need for the concurrent assessment of the sources of regret.

- In line with the original conceptualization of regret by Loomes and Sugden (1982) and following Sugden's (1985) work (as detailed in the Conceptual and Theoretical Framework section above), we have been able to show to that the comparison of 'what is' with 'what might have been' is applicable to both the decision process and the product. implications:

In the addition to the above theoretical contributions our research also proposes the following practical

- $\quad$ The distinction between the sources of regret could ameliorate the mis-attributions that consumers make subsequent to an unfavorable outcome. For instance, when regret arises due to the decision-making process one might realize one's own mistake and consequently not blame or spread negative word-of-mouth regarding the marketer. The problem of mis-attribution is further exacerbated when consumers simply 
switch and the firm steadily loses its customer base. A careful attribution is not always performed subsequent to an unfavorable outcome (Soscia 2007). Hence, firms might look into increasing consumer responsibility by providing genuine and honest information that will be helpful during the decision-making process.

- $\quad$ The research also indicates that both experts and non-experts regret the product equally when the product is unfavorable. This will lead most consumers to assume their particular model or even the entire manufacturer has poor quality. The consumer is at that point using the Law of Small Numbers (Tversky and Kahneman 1974). The marketer needs to promote facts about the population to diminish the salient personal experience. For example, by advertising that the product is highly rated in reliability, the marketer reduces the amount of regret experienced by the consumer. This allows the consumer to see that their failed product was an aberration and that product may still be a valid option.

- $\quad$ Results indicated that experts understand that the decision-making process was effective, even when the product fails. As mentioned earlier, Ratner and Herbst (2005) have shown that emotional reactions, subsequent to unfavorable outcomes, sometimes lead individuals to adopt less than optimal decision the next time, even though the foregone decision has a better chance of producing a better outcome. Marketers need to battle this phenomenon by re-affirming the decision-making process of the consumer. They can reaffirm the decision-making process by showing the consumer base-rate information (Dickson 1982). For example, the marketer needs to advertise that their product won JD Power's Award for Highest Customer Satisfaction. This ought to re-affirm the consumer that the decision-making process was optimal and they should use the same process next time.

Several avenues for future research emerge from this study when the limitations of the same are accepted. First, the mechanism for the segregation of the sources of regret was limited only to the level of expertise of the consumers; other variables should be tested to obtain more valid results. Second, behavioral intentions should be studied in order to get a better idea of the practicality of our conceptualization. Third, although regret was the main variable of interest here, other equally important variables, like disappointment, might be measured to get a broader view of human emotions and their implications. Finally, we used students as subjects and a car as the product. Replication using non-student subjects and a different product may lend a considerable degree of external validity.

\section{CONCLUSION}

A concurrent examination of the sources of regret has been proposed in order to better understand the nature and experience of the emotion. Based on the initial conceptualization of the emotion by Loomes and Sugden (1982), we developed two sources of regret - regret from the decision-making process and regret from product. Specifically, the underlying emphasis for each source is the comparative process of 'what is' with 'what might have been'. While extant regret research in marketing has essentially examined and measured regret either from the decision process or from the product, Das and Kerr (2010) presented a conceptual investigation of the concurrent examination of the sources of regret. To the best of our knowledge, the current research is the first in the domain of marketing to empirically implement and investigate the concurrent examination of the sources of regret. Using the moderator of consumer expertise, results from the study indicate that non-experts, compared to experts, were significantly less likely to recognize (and hence experience greater regret from) an optimal decision process when the product purchased was unfavorable. However, the significant difference in greater regret was evidenced when measuring regret from the decision process, but not when measuring regret from the product.

\section{AUTHOR INFORMATION}

Neel Das is an Assistant Professor of Marketing at Appalachian State University. He earned his PhD from Louisiana State University, Baton Rouge, LA in 2004. He has published in journals such as Journal of Applied Business Research, Journal of Marketing Theory and Practice, and Journal of Advertising. E-mail: dasn@appstate.edu. Corresponding author.

Brad Joffe finished his Bachelor's degree in Marketing summa cum laude from Appalachian State University, Boone, NC in December 2010. He is now working at Citco Fund Services Inc., in Charlotte, NC as a data analyst. E-mail: bjoffe@citco.com 


\section{REFERENCE}

1. Abenroth, Lisa J. and Kristin Diehl (2006). "Now or Never: Effects of Limited Purchase Opportunities on Patterns of Regret over Time", Journal of Consumer Research, 33 (December), 342 - 351.

2. Bolton Ruth N., P. K. Kannan and Matthew D. Bramlett (2000). "Implication of Loyalty Program Membership and Service Experiences for Customer Retention and Value", Journal of The Academy of Marketing Science, 28(1), 95 - 108.

3. Brucks, M. (1985). “The Effects Of Product Class Knowledge On Information Search Behavior”, Journal of Consumer Research, June, $1-16$.

4. Bui, My, Anjala S. Krishen, and Kenneth Bates (2011). "Modeling Regret Effects on Consumer PostPurchase Decisions", European Journal of Marketing, 45 (7/8), 1068 - 1090.

5. Cai, Yi and Brenda Cude (2011). "Reference Prices and Consumers' Feeling of Regret: An Investigation of Consumers' Use of an Online Price-Bidding Method", International Journal of Consumer Studies, 35, 441 $-447$.

6. Connolly, Terry and Marcel Zeelenberg (2002). "Regret in Decision Making", Current Directions in Psychological Science, 11(6), December, 212 - 216.

7. Das, Neel and Anthony H. Kerr (2010). "Woulda, Coulda, Shoulda: A Conceptual Examination of the Sources of Postpurchase Regret", Journal of Marketing Theory and Practice, Spring, 18(2), 171 - 180.

8. Dickson, Peter R. (1982). "The Impact of Enriching Case and Statistical Information on Consumer Judgments", Journal of Consumer Research, March, 398 - 408.

9. Gilovich, Thomas and Victoria. H. Medvec (1995). "The Experience of Regret: What, When and Why," Psychological Review, 102, 379 - 395.

10. Hastie, Reid and Robyn M. Dawes (2001). "Rational Choice in an Uncertain World: The Psychology of Judgment and Decision Making", Sage Publications Inc.

11. Heitmann, Mark, Donald R. Lehmann and Andreas Herrmann (2007). "Choice Goal Attainment And Decision And Consumption Satisfaction”, Journal of Marketing Research, 44(2), 234-250.

12. Higgins, E. Tory (2000). "Making a good decision: Value from fit", American Psychologist, 55, 1217 1230.

13. Janis, I. and L. Mann (1977). "Decision-Making: A Psychological Analysis of Conflict, Choice, and Commitment", New York: The Free Press

14. Higgins, E. Tory, Christopher J. Camacho, Lorraine C. Idson, Scott Spiegel, and Abigail A. Scholer (2008). "How Making the Same Decision in a "Proper Way" Creates Value", Social Cognition, 26 (5), 496 - 514.

15. Inman, Jeffrey J., James S. Dyer and Jianmin Jia (1997). "A Generalized Utility Model Of Disappointment And Regret Effects On Post-Choice Valuation,” Marketing Science, 16(2), 97 - 111.

16. Kahneman, Daniel and Amos Tversky (1979). "Prospect Theory: An Analysis of Decision Under Risk", Econometrica, 47, 263 - 291.

17. Kahneman, Daniel and Amos Tversky (1982). "The Psychology of Preferences”, Scientific American, 246, $160-173$.

18. Landman, Janet (1993). "Regret: The Persistence of The Possible", New York, Oxford University Press.

19. Loomes, Graham and Robert Sugden (1982). "Regret Theory: An Alternative Theory of Rational Choice Under Uncertainty", The Economic Journal, 92 (December), 805 - 824.

20. Mitchell, Andrew A. and Peter A. Dacin (1996). "The Assessment of Alternative Measures of Consumer Expertise", Journal of Consumer Research, 23 (December), 219 - 239.

21. Ratner, R. K. and K. C. Herbst (2005). "When Good Decision have Bad Outcomes: The Impact of Affect on Switching Behavior", Organizational Behavior and Human Decision Processes, 96, 23 - 37.

22. Shanteau, James (1992). "The Psychology of Experts: An Alternative View", Expertise and Decision Support, in G. Wright and F. Bolger (Eds.), Plenum Press, NY, $11-23$.

23. Shimanoff, Susan B. (1984). "Commonly Named Emotions in Everyday Conversations", Perceptual and Motor Skills, 58 (April), 514.

24. Soscia, Isabella (2007). "Gratitude, Delight, or Guilt: The Role of Consumers' Emotions in Predicting Postconsumption Behaviors", Psychology and Marketing, 24(10), October, 871 - 894.

25. Sugden, Robert (1985). "Regret, Recrimination and Rationality", Theory and Decision, 19 (July), 77 - 99. 
26. Syam, Niladri, Partha Krishnamurthy and James D. Hess (2008). "That's What I Thought I Wanted? Miswanting and Regret for a Standard Good in a Mass Customized World”, Marketing Science, 27(3), May-June, 379 - 397.

27. Taylor, Kimberly (1997). “A Regret Theory Approach to Assessing Consumer Satisfaction”, Marketing Letters, 8(2), 229 - 238.

28. Tsiros, Michael and Vikas Mittal (2000). "Regret: A Model of Its Antecedents and Consequences in Consumer Decision Making," Journal of Consumer Research, 26 (June), 401 - 417.

29. Tversky, Amos and Daniel Kahneman (1971). "Belief in the Law of Small Numbers", Psychological Bulletin, August, $105-110$.

30. Yates, J. Frank and Michael D. Tschirhart (2006). "Decision-Making Expertise," in The Cambridge Handbook of Expertise and Expert Performance, edited by K. Anders Ericsson, Neil Charness, Paul J. Feltovich, and Robert R. Hoffman, Cambridge University Press, 421 - 438.

31. Zeelenberg, Marcel (1999). "The Use of Crying Over Spilled Milk: A Note on the Rationality and Functionality of Regret”, Philosophical Psychology, 12(3), 325 - 340.

32. Zeelenberg, Marcel and Rik Pieters (2007). "A Theory of Regret Regulation”, Journal of Consumer Psychology, 17(1), 3 - 18 .

33. Zeeleberg, Marcel, W. W. Van Dijk, and A. S. R. Manstead (1998). "Reconsidering the Relationship between Regret and Responsibility”, Organizational Behavior and Human Decision Processes, 74, 254 272 .

34. Zeeleberg, Marcel, W. W. Van Dijk, and A. S. R. Manstead (1998). "Regret and Responsibility Resolved? Evaluating Ordonez and Connolly's (2000) Conclusions”, Organizational Behavior and Human Decision Processes, 81, 143 - 154.

35. Zeeleberg, Marcel, Eric van Dijk, Kees van den Bos and Rik Pieters (2002). "The Inaction Effect in the Psychology of Regret," Journal of Personality and Social Psychology, 82(3), 314 - 327.

36. Zeelenberg, Marcel and Rik Pieters (2004). "Beyond valence in customer dissatisfaction: A review and new findings on behavioral responses to regret and disappointment in failed services", Journal of Business Research, 57, 445 - 455. 